\title{
Parsonage-Turner syndrome presenting with left shoulder pain and weakness: a case report
}

\author{
Fazıl Kulaklı®, Sencer Duman²๑
}

${ }^{1}$ Department of Physical Medicine and Rehabilitation, Giresun University, Prof. Dr. A. İlhan Özdemir Training and Research Hospital, Giresun, Turkey

${ }^{2}$ Department of Neurosurgery, Bulancak State Hospital, Giresun, Turkey

\begin{abstract}
Parsonage-Turner syndrome is a clinical syndrome that begins with a sudden, neuropathic pain on the shoulder girdle, followed by pain loss over time. It is a rare syndrome that can be overlooked during differential diagnosis of shoulder pain and weakness. In this paper, we aimed to present a 39-year-old male patient with sudden onset left shoulder pain and weakness who were diagnosed with Parsonage-Turner syndrome.

Keywords: Parsonage-Turner syndrome, shoulder pain, weakness
\end{abstract}

$\mathbf{P}$ arsonage-Turner syndrome (PTS) was first demonstrated by Parsonage and Turner in 1948. However, the syndrome has different names such as acute brachial neuropathy, acute brachial plexitis and idiopathic brachial plexopathy [1]. It is a clinical syndrome characterized by sudden onset, severe and mostly neuropathic charactershoulder pain and subsequently developing muscle weakness [2]. PTS frequently confused with shoulder and neck pathologies and the syndrome must be considered during the differential diagnosis of patients presenting with shoulder pain and weakness [3]. The frequency of PTS is known as $1.64 / 100,000$ and more common in 20-60 age male population and is a treatable disease with good prognosis [2]. The aim of presenting this case report is to raise awareness of PTS and emphasize that it should be included in the differential diagnosis of shoulder pain and upper extremity weakness.

\section{CASE PRESENTATION}

A 39-year-old male patient was admitted to our outpatient clinic with complaints of pain and weaknessonhis left shoulder. A sudden onset of left shoulder pain occured one month ago and weakness of shoulder added to pain in the following few days. Patient complained of numbness of left hand at the same time and applied to orthopedics department with these complaints. The patient was diagnosed with muscle rupture and the necessary drugs were started but there was no improvement onhis complaints. There were no history of trauma, infection or neck painand he did not describe another systemic disease. When the patient was questioned neurologically, it was understood that there was no neurological problem in addition to the current situation. Physical examination showed atrophy of the left deltoid muscle. The range of motion of the upper left limb joints was normal. His elbow flexion was $2 / 5$, extension $2 / 5$, wrist dorsiflexion was $2 / 5$ and finger movements were $5 / 5$. Hypoesthesia was detected on the lateral side of the left arm and forearm. The left biceps-brachioradial and triceps reflexes were decreased and there was no pathological reflex. The other extremities and cranial system were normal. No pathology was found in the brain-cervical-brachial 


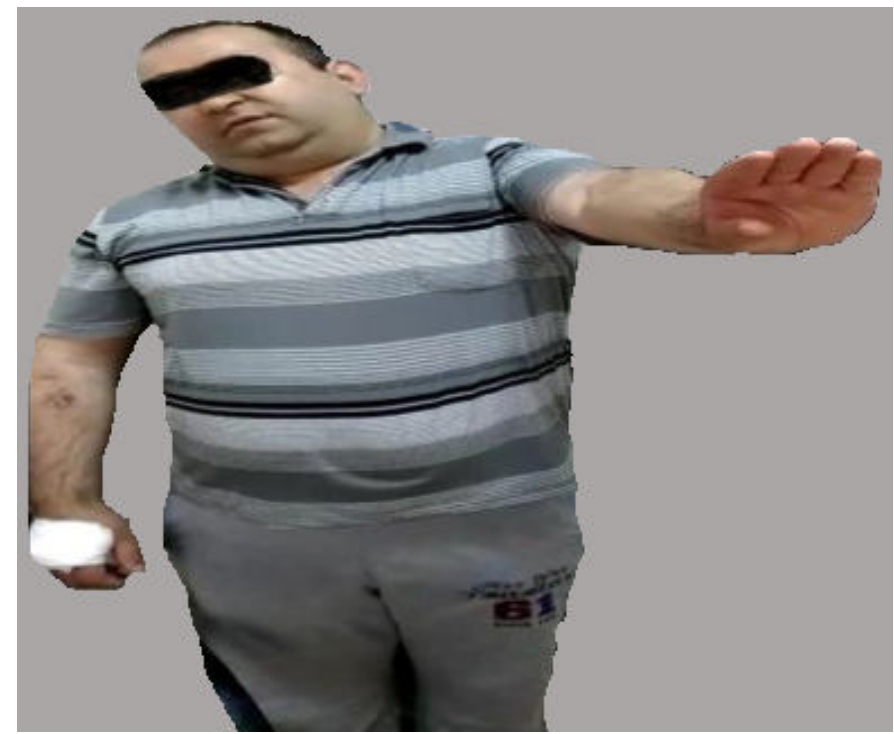

Fig. 1. 2/5 shoulder flexion muscle strength at the second week of rehabilitation program

plexus and shoulder magnetic resonance imagingand no pathology was found in the laboratory tests of the patient. In the electroneuromyography, the patient was found to have plexopathy in the subacute process in the upper-middle trunk of the brachial plexus. Chest $\mathrm{X}$-ray required suspicion of possible lung diseases, doubtful consolidation was detected and the patient consultated to chest diseases. A thoracic computerized tomography (CT) scan was recommended and the result of CT was reported as normal. In the light of these findings, the patient was diagnosed as PTS and $40 \mathrm{mg}$ methylprednisolone treatment withexercise program were initiated. After using methylprednisolone and 30 sessions of exercise program, the patient's pain and muscle strength improved andhe was discharged with full recovery (Figs. 1 and 2).

\section{DISCUSSION}

Shoulder pain is a significant part of the patients admitted to the physical medicine and rehabilitation outpatient clinic. Although there are many intrinsic and extrinsic causes of shoulder pain, the most common cause is known as subacromial impingement syndrome.In cases where shoulder pain is accompanied by weakness, brachial plexus, cervical roots, spinal cord and cranial system pathologies should be considered in the differential diagnosis.In this respect, PTS, which is a treatable disease with good prognosismay

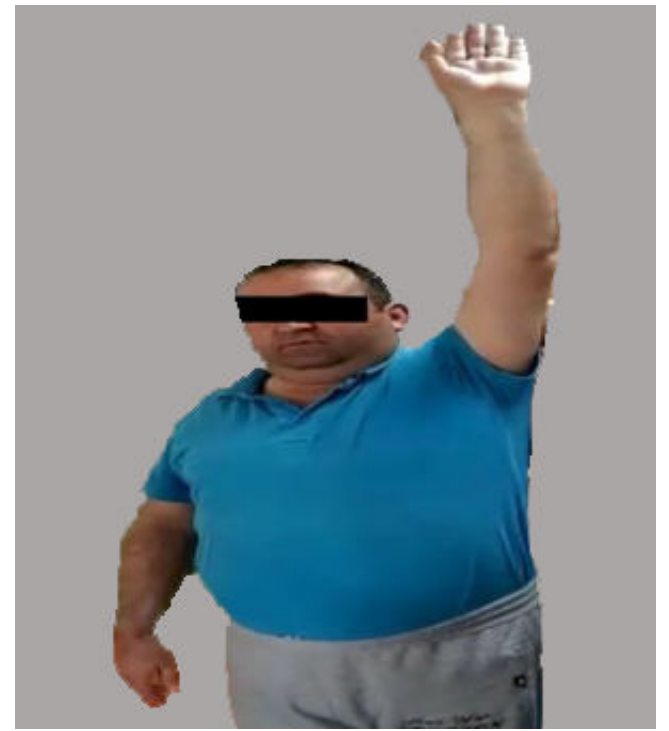

Fig. 2. 5/5 shoulder flexion muscle strength at the end of the rehabilitation program.

be seen as an important syndrome to be considered in the differential diagnosis of shoulder pain and weakness. The frequency of PTS is known as 1.64/100,000 and more common in 20-60 age range male population [2]. The most common cause of brachial plexopathies is known as trauma. However, PTS was pointed out asthe most common cause of brachial plexopathies in a study evaluating 203 cases [4]. There was no difference in terms of frequency of involvement between the right and left extremities and no correlation was found for the dominant hand. Involvement is usually unilateral but bilateral involvement may be seen as $1 / 3$ ratio $[5,6]$. The cause of PTS is not known yet. Although viral infections (influenza-B, parvovirus, HIV), vaccines, autoimmune causes, heavy exercise, previous operations and hereditary causes have been blamed, the etiology has not been elucidated [7-9]. Although there was no story of heavy exercise in our case, it was thought that the shoulder might beexposed to continuous microtrauma due to the profession of cookery. As in our case, laboratory tests(complete blood count, erythrocyte sedimentation rate, serum electrolytes, liver function tests, urine analysis, immunological studies) in PTS patients are generally normal. Cervical vertebrae and shoulder radiographs are usually normal in PTS patients. Shoulder pathologies which may cause pain and weakness such as impingement syndrome, adhesive capsulitis, etc. may be frequently confused and disregarded. In our case, noshoulder-related pathologywas found. In the differ- 
ential diagnosis; cervical disc herniations, poliomyelitis, amyotrophic lateral sclerosis, herpes zoster, spinal cord or brachial plexus tumors and traumatic, compressive nerve injuries, myopathies, polymyalgia rheumatica and other rheumatologic diseases should also be considered [5]. The most useful study for diagnosis isindicated as electromyography. Although the electromyographic findings may be variable, the most important feature is the presence of acute denervation findings showing axonal neuropathy such as fibrillation and positive sharp waves [10]. Treatment of PTS is symptomatic and usually prognosis is good. The goals of treatment are determined as relieving pain, maintaining range of motion of the joints, restoring muscle strength and maximizing functional capacity of patient. There is no evidence of the superiority of any treatment. Corticosteroids and intravenous immunoglobulin in the early period of weakness has been suggested to prevent progression of disease, but its effect has not been proven [11].In our case, we applied methylprednisolone as pharmacologic treatment and physical medicine and rehabilitation program as nonpharmacologic treatment. As a result of the treatments, the patient's muscle strength was completely improved and the pain completely passed. Our case is similar to other cases in the literature but the importance of this case is its rarity. In addition the diagnosis of PTS was not considered enough in the differential diagnosis of shoulder pain and weakness as mentioned above. Therefore it is aimed to raise awareness of PTS in this case report.

\section{CONCLUSION}

PTS should be considered in the differential diagnosis of shoulder pain and weakness. Providing adequate information to the patient about the good prognosis of the disease will allow the treatment to proceed more positively. Although a special rehabilitation program is the most important parameter, we believe that corticosteroid therapy will be beneficial.

\section{Informed consent}

Written informed consent was obtained from the patient for publication of this case report and any accompanying images.

\section{Conflict of interest}

The authors declared that there are no potential conflicts of interest with respect to the research, authorship, and/or publication of this article.

\section{REFERENCES}

1. Sathasivam S, Lecky B, Manohar R, Selvan A. Neuralgic amyotrophy. J Bone Joint Surg Br 2008;90:550-3.

2. Şahin E, Şenocak Ö, Bacakoğlu AK, Öztura İ, Gözüm M, Peker Ö. [Neuralgic amyotrophy as the primary cause of shoulder pain in a patient with rotator cuff tear]. Acta Orthop Traumatol Turc 2009;43:190-2. [Article in Turkish]

3. Pessa ME, Verriello L, Valente M, Gigli GL. A rare case of pure sensitive Parsonage-Turner syndrome. Neurol Sci 2019;40:1499-501.

4. Moghekar AR, Moghekar AR, Karli N, Chaudhry V. Brachial plexopathies: etiology, frequency, and electrodiagnostic localization. J Clin Neuromuscul Dis 2007;9:243-7.

5. Koca İ, İbaş E, Karagüllü H, Altındağ O, Gür A. [The case of neuralgic amyotrophy with bilateral axillary nevre involvement]. Turk J Phys Med Rehabil 2011;57:107-10. [Article in Turkish] 6. Ohta R, Shimabukuro A. Parsonage-Turner syndrome in a patient with bilateral shoulder pain: a case report. J Rural Med 2017;12:135-8.

7. England JD, Sumner AJ. Neuralgic amyotrophy: an increasingly diverse entity. Muscle Nerve 1987;10:60-8.

8. Dumitru D. Brachial plexopathies and proximal mononeuropathies. Electrodiagnostic Medicine. 1st ed. Philadelphia: Hanley (Belfus); 1995: p.585-642.

9. Watts GD, O'Briant KC, Chance PF. Evidence of a founder effect and refinement of the hereditary neuralgic amyotrophy (HNA) locus on $17 \mathrm{q} 25$ in American families. Hum Genet 2002;110:166-72.

10. Nardone R, Benhart H, Pozzera A, Taddei M, Tezzon F. Respiratory weakness in neuralgic amyotrophy: report of two cases with phrenic nerve involvement. Neurol Sci 2000;21:177-81. 11. Rubin DI. Neuralgic amyotrophy: clinical features and diagnostic evaluation. Neurologist 2001;7:350-6. 